\title{
Classical stereodynamics in Ar + NO inelastic collisions $\dagger$
}

\author{
F. J. Aoiz, ${ }^{a}{ }^{a}$ V. J. Herrero, ${ }^{b}$ V. Sáez Rábanos ${ }^{c}$ and J. E. Verdasco ${ }^{d}$ \\ ${ }^{a}$ Departamento de Química Física, Facultad de Química, Universidad Complutense, \\ 28040 Madrid, Spain \\ ${ }^{b}$ Instituto de Estructura de la Materia (CSIC), Serrano 12328006 Madrid, Spain \\ ${ }^{c}$ Departamento de Química y Bioquímica, ETSI Montes UPM 28040 Madrid, Spain \\ ${ }^{d}$ Departamento de Química Física, Facultad de Química, Universidad Complutense, \\ 28040 Madrid, Spain
}

Received 24th June 2004, Accepted 14th July 2004

First published as an Advance Article on the web 30th July 2004

\begin{abstract}
The stereodynamics of the $\mathrm{Ar}+\mathrm{NO}(j=0)$ rotational inelastic excitation has been investigated at $66 \mathrm{meV}$ by means of quasiclassical trajectories on a recent $a b$ initio potential energy surface. A marked correlation between the preferred sense of rotation of NO and the scattering plane is obtained for the highest rotational levels accessible, which are excited in strong repulsive collisions. This result is in qualitative agreement with recent quantum mechanical calculations and experimental measurements. For the lower rotational levels, where the interactions are not so repulsive, the preferred sense of rotation is found to oscillate with scattering angle, but the intensity of the oscillations is small and their angular range is not entirely coincident with those from quantum mechanics and experiment. Classical dynamics, even including attractive interactions, cannot account properly for the mentioned oscillatory behaviour. Secondary encounters between the outgoing Ar atom and NO molecule, giving rise to 'chattering', are found to be relatively frequent, leading to a decrease in the final rotational energy of $\mathrm{NO}$ with respect to that attained in the first encounter. Chattering trajectories are defined and their mechanism is characterized.
\end{abstract}

\section{Introduction}

As pointed out since the seventies by Herschbach and coworkers $^{1-4}$ the correlations between the vector quantities (relative velocities and angular momenta) characterizing collisions between atoms and molecules provide a unique insight into the dynamical properties of these systems. The simplest correlations are those between pairs of vectors. Among them the most familiar one is that between the velocity vectors before $\boldsymbol{k}$ and after $\boldsymbol{k}^{\prime}$ the collision, which corresponds to the differential cross section (DCS).

Three and four vector correlations carry also valuable information about the details of atom-diatom collisions, especially those relating the various angular momenta (rotational or orbital before or after the collision) to the $\boldsymbol{k}-\boldsymbol{k}^{\prime}$ plane. However the dynamical implications of these correlations are not so straightforward, and their experimental determination is difficult.

The theoretical formalism for the study of vector correlations in molecular collisions was developed over the nineties by various groups that applied both quasiclassical trajectories (QCT) and quantum-mechanical (QM) methods to derive relevant stereodynamical properties susceptible of experimental investigation. ${ }^{5-12}$ Miranda et al. ${ }^{13}$ presented a unified QMQCT treatment and demonstrated the equivalence between the two approaches. In parallel with these theoretical progress, different experimental schemes based on the use of polarized lasers, were implemented in various laboratories and yielded a wealth of data on the stereodynamics of different elementary collision processes. ${ }^{14-29}$

In recent works ${ }^{30,31}$ rotational orientation and alignment in the inelastic $\mathrm{NO}+$ Ar scattering have been experimentally

$\dagger$ Electronic supplementary information (ESI) available: Fig. 3 in colour. See http://www.rsc.org/suppdata/cp/b4/b409607j/ investigated at a collision energy of $\approx 66 \mathrm{meV}$ with a combination of polarized lasers, multiphoton ionization probing and velocity-mapped ion imaging detection. Wade et al. ${ }^{31}$ have measured the collisional-induced alignment of the rotational angular momentum of $\mathrm{NO}$ and have found good agreement between the experimental alignment moments for low rotational excitation and the results of theoretical calculations performed on the CCSD(T) potential energy surface (PES) of Alexander. ${ }^{32,33}$ For the higher excited levels the agreement deteriorates. The authors of this work have stressed the importance of taking into account alignment effects in order to derive the proper differential cross sections from the experimental data. On the other hand, Lorenz et al. have reported a direct measurement of the preferred sense of NO rotation after collision with Ar. This was the first experimental observation of molecular rotational orientation in individual bimolecular collisions. The sense of rotation of the inelastically scattered NO molecules was found to vary with deflection angle and to be strongly dependent on the final rotational state. For the lower $j^{\prime}$ values the sense of orientation was seen to oscillate with deflection angle, whereas for the highest NO $j^{\prime}$ states allowed, a marked preference for a given sense of rotation was obtained. Quantum calculations on the mentioned $\operatorname{CCSD}(\mathrm{T})$ $\mathrm{PES}^{32,33}$ could account for the angular behaviour of the sense of rotation just described. Classical calculations using a simple potential model in which the NO molecule is taken as a rigid ellipsoid, suggested that multiple-encounter ("chattering") collisions could play an important role in the defined sense of rotation observed in the highest $j^{\prime}$ levels, but could not account for the alternating sense of rotation observed in the lower ones. Neglect of the attractive part of the potential or absence of interferences (e.g. between direct and "chattering" collisions leading to the same $j^{\prime}$ ) in the classical model were suggested as possible causes of the failure to reproduce the oscillating behaviour. 
These findings and the availability of an accurate potential energy surface for $\mathrm{Ar}+\mathrm{NO}$ in the range of energies relevant to the experiments ${ }^{32,33}$ provide a strong motivation for a detailed QCT investigation of the stereodynamics of $\mathrm{Ar}+$ NO inelastic collisions specially if one considers the equivalence between the QM and QCT formulations mentioned above $^{13}$ and the good dynamical insight provided by the classical approach. In this work we present a global QCT study of the most relevant vector correlations for this system. The results are discussed and compared to the recent experimental and QM data on the rotational orientation of $\mathrm{NO}$ after collision with Ar.

\section{Method}

The QCT calculation method is essentially the same as used in a previous work on this system; ${ }^{34}$ it is described in various $\operatorname{articles}^{35-37}$ and only the specific details pertaining to the present study will be given here. A total number of 470000 trajectories have been calculated on the $V_{\text {sum }}$ PES of Alexan$\mathrm{der}^{32}$ for a collision energy, $E_{\mathrm{col}}$, of $532 \mathrm{~cm}^{-1}(66 \mathrm{meV})$ and for the $v=0, j=0$ internal state of $\operatorname{NO}(\Omega=1 / 2)$. The $V_{\text {sum }}$ surface, which is the form convenient for the description of the adiabatic $(\Delta \Omega=0)$ nuclear motion, is defined as: ${ }^{38}$

$$
V_{\text {sum }}(R ; \vartheta)=0.5\left[V_{\mathrm{A}^{\prime \prime}}(R, \vartheta)+V_{\mathrm{A}^{\prime}}(R, \vartheta)\right]
$$

where $R$ is the distance between the atom and the center of mass of the molecule and $\vartheta$ is the angle between $R$ and the direction of the internuclear distance, $r$. The subindices $\mathrm{A}^{\prime}$ and $\mathrm{A}^{\prime \prime}$ correspond to the two lowest $\operatorname{CCSD}(\mathrm{T})$ ab initio electronic states of the ArNO system. ${ }^{32}$ During the integration of the classical equations of motion, the rigid rotor constraint $(r=$ constant) for $\mathrm{NO}$ was introduced by the method of Lagrange multipliers. ${ }^{34,39,40}$ For the assignment of the final rotational quantum number, $j^{\prime}$, of NO the square of the classical angular momentum, $j^{\prime 2}$, is equated to $j^{\prime}\left(j^{\prime}+1\right) / \hbar^{2}$. The values of $j^{\prime}$ thus obtained are then rounded to the nearest integer.

In order to determine the value of the maximum impact parameter, $b_{\max }$, to be used in the calculations, the change in rotational quantum number $\Delta j$ with impact parameter was monitored. The impact parameter was increased until no trajectories leading to $\Delta j>0.5$ were found. With this procedure a value of $6.3 \AA$ was derived for $b_{\max }$.

The reference frame we will use throughout this paper is that in which the $z$ axis is parallel to the initial relative velocity vector, $\boldsymbol{k}$, and $x z$ is the scattering plane containing the initial and final relative velocity vectors $\boldsymbol{k}, \boldsymbol{k}^{\prime}$ with the $y$ axis in the $\boldsymbol{k} \times \boldsymbol{k}^{\prime}$ direction; see Fig. 1 . In this frame $\theta$ is the scattering angle (i.e. the angle between $\boldsymbol{k}$ and $\boldsymbol{k}^{\prime}$ ) and $\theta_{j^{\prime}}$ and $\phi_{j^{\prime}}$ are the polar and azimuthal angles of the final rotational angular momentum, $\boldsymbol{j}^{\prime}$, and the azimuthal angle of the initial orbital angular momentum, $\boldsymbol{l}$, is $\phi_{l}$. The angle between $\boldsymbol{l}$ and $\boldsymbol{j}^{\prime}$ is $\theta_{l j^{\prime}}$.

The classical description of polarized angular momenta is the joint probability density function (PDF), $P\left(\theta, \theta_{j^{\prime}}, \phi_{j^{\prime}}\right)$, whose value gives the relative probability of inelastic scattering into an angle $\theta$, when the $\boldsymbol{j}^{\prime}$ vector points along the direction defined by the $\theta_{j^{\prime}}$ and $\phi_{j^{\prime}}$ angles. This function can be expanded in terms of complex conjugate of the modified spherical harmonics. ${ }^{13}$ The expansion reads

$$
P\left(\theta, \theta_{j^{\prime}}, \phi_{j^{\prime}}\right)=\sum_{k=0}^{\infty} \sum_{q=-k}^{k} \frac{2 k+1}{4 \pi}\left(\frac{2 \pi}{\sigma} \frac{\mathrm{d} \sigma_{k q}}{\mathrm{~d} \omega}\right) C_{k q}^{*}\left(\theta_{j^{\prime}}, \phi_{j^{\prime}}\right)
$$

where the $\theta$-dependent expansion coefficients, $(2 \pi / \sigma)\left(\mathrm{d} \sigma_{k q} / \mathrm{d} \omega\right)$, are the polarization dependent differential cross sections, PDDCS. In particular, the $(2 \pi / \sigma)\left(\mathrm{d} \sigma_{00} / \mathrm{d} \omega\right)$ PDDCS is the flux normalized differential cross section.

By integration of this equation over all scattering angles, we obtain the classical probability density function of finding the
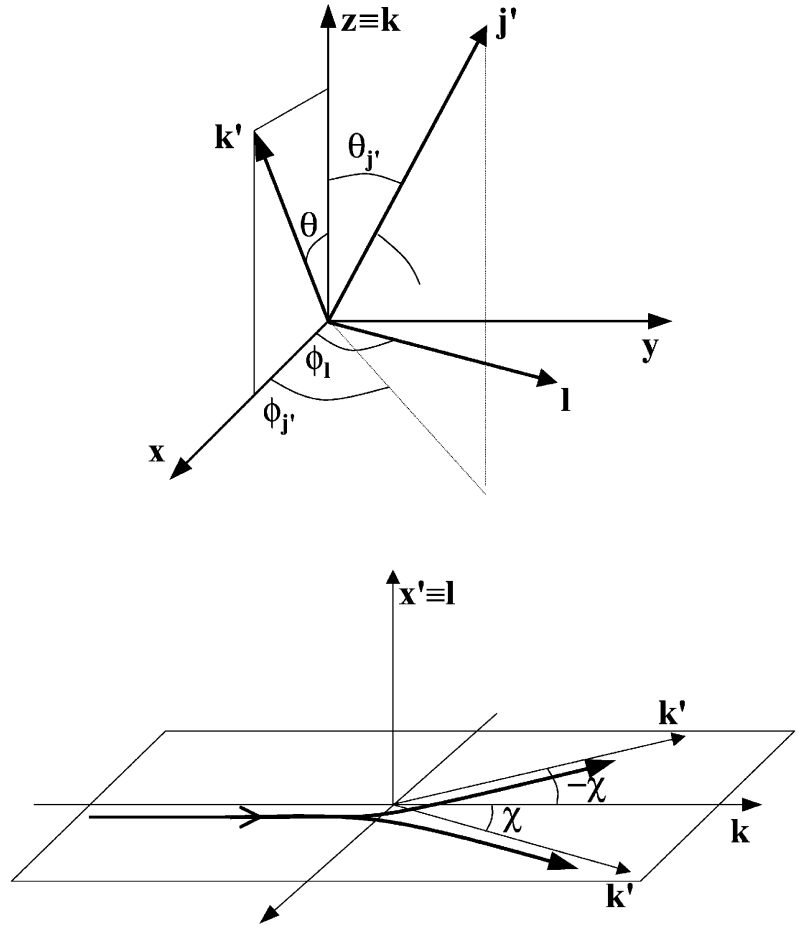

Fig. 1 Top: Diagram of the $\boldsymbol{k}-\boldsymbol{k}^{\prime}$ scattering frame showing the relevant angles in the various vector correlations. The scattering angle is $\theta$ and the direction of the product rotational angular momentum are given in this frame by the angles $\theta_{j^{\prime}}, \phi_{j^{\prime}}$, and that of the orbital angular momentum by $\phi_{l}$. Bottom: Diagram showing two ideal coplanar trajectories in which the scattering plane $\boldsymbol{k}-\boldsymbol{k}^{\prime}$ is represented with respect to the direction of the orbital angular momentum, $\boldsymbol{l}$. If the value of $\phi_{l}$ is known it is possible to discern between trajectories with positive and negative deflection angles, $\chi$, for the same value of the scattering angle $(\theta=|\chi|)$.

vector $\boldsymbol{j}^{\prime}$ pointing along the direction specified by the $\theta_{j^{\prime}}, \phi_{j^{\prime}}$ angles, integrated over the scattering angle, $P\left(\theta_{j^{\prime}}, \phi_{j^{\prime}}\right)$.

$$
P\left(\theta_{j^{\prime}}, \phi_{j^{\prime}}\right)=\sum_{k=0}^{\infty} \sum_{q=-k}^{k} \frac{2 k+1}{4 \pi} a_{q}^{(k)} C_{k q}^{*}\left(\theta_{j^{\prime}}, \phi_{j^{\prime}}\right)
$$

where the $\theta$ independent polarization parameters, $a_{q}{ }^{(k)}$, are given by

$$
a_{q}^{(k)}=\int_{-1}^{1} \frac{2 \pi}{\sigma} \frac{\mathrm{d} \sigma_{k q}}{\mathrm{~d} \omega} \mathrm{d} \cos \theta
$$

Another distribution function to be considered is the conditional probability density function, $P\left(\theta_{j^{\prime}}, \phi_{j^{\prime}} \mid \theta\right)$, which gives the relative probability of finding the $\boldsymbol{j}^{\prime}$ vector along the direction specified by the $\theta_{j^{\prime}}$ and $\phi_{j^{\prime}}$ angles when the scattering is at angle $\theta .{ }^{5,13}$ It can be written as:

$$
P\left(\theta_{j^{\prime}}, \phi_{j^{\prime}} \mid \theta\right)=\sum_{k=0}^{\infty} \sum_{q=-k}^{k} \frac{2 k+1}{4 \pi} \frac{\mathrm{d} \sigma_{k q} / \mathrm{d} \omega}{\mathrm{d} \sigma_{00} / \mathrm{d} \omega} C_{k q}^{*}\left(\theta_{j^{\prime}}, \phi_{j^{\prime}}\right)
$$

These expansion coefficients, the renormalized PDDCSs, are given by:

$$
\begin{aligned}
\rho_{q}^{\{k\}} & \equiv \frac{\mathrm{d} \sigma_{k q} / \mathrm{d} \omega}{\mathrm{d} \sigma_{00} / \mathrm{d} \omega} \\
& =\int_{0}^{2 \pi} \int_{-1}^{1} P\left(\theta_{j^{\prime}}, \phi_{j^{\prime}} \mid \theta\right) C_{k q}\left(\theta_{j^{\prime}}, \phi_{j^{\prime}}\right) \mathrm{d} \cos \left(\theta_{j^{\prime}}\right) \mathrm{d} \phi_{j^{\prime}}
\end{aligned}
$$

Therefore they are the conditional expectation values of $C_{k q}\left(\theta_{j^{\prime}}, \phi_{j^{\prime}}\right)$ at the scattering angle $\theta$.

Because all these expansion coefficients (the PDDCSs, the polarization parameters and the renormalized PDDCSs) are, in general, complex quantities we have used the Hertel-Stoll 
convention ${ }^{13}$ to define the corresponding real expansion coefficients, $a_{q \pm}^{\{k\}}$ :

$$
\begin{gathered}
a_{q+}^{\{k\}}=\frac{1}{\sqrt{2}}\left[(-1)^{q} a_{q}^{(k)}+a_{-q}^{(k)}\right] \\
a_{q-}^{\{k\}}=\frac{1}{\mathrm{i} \sqrt{2}}\left[(-1)^{q} a_{q}^{(k)}-a_{-q}^{(k)}\right] \\
a_{0}{ }^{\{k\}}=a_{0}{ }^{\{k\}}
\end{gathered}
$$

with similar expressions for the real PDDCSs and renormalized PDDCSs.

For nonchiral systems, the nonvanishing real polarization parameters and PDDCSs are: (i) with $k$ odd and component $q-$, and (ii) with $k$ even and component either zero or $q+$.

To calculate these stereodynamical parameters, we remind that the real PDDCSs can be expressed as a series of modified spherical harmonics: 5,13

$$
\frac{2 \pi}{\sigma} \frac{\mathrm{d} \sigma_{k q \pm}}{\mathrm{d} \omega}=\frac{1}{2} \sum_{k_{1} \geq q}\left(2 k_{1}+1\right) s_{\{k\} q \pm}^{k_{1}} C_{k_{1}-q}\left(\theta_{j^{\prime}}, 0\right)
$$

where the coefficients $s_{\{k\} q \pm}^{k_{1}}$ are given by

$$
\begin{aligned}
s_{\{k\} q+}^{k_{1}}= & \sqrt{2}\left\langle C_{k_{1} q}(\theta, 0) C_{k q}\left(\theta_{j^{\prime}}, 0\right) \cos \left(q \phi_{j^{\prime}}\right)\right\rangle \\
& =\sqrt{2} \frac{1}{N_{j^{\prime}}} \sum_{i=1}^{N_{j^{\prime}}} C_{k_{1} q}\left(\theta^{(i)}, 0\right) C_{k q}\left(\theta_{j^{\prime}}^{(i)}, 0\right) \cos \left(q \phi_{j^{\prime}}^{(i)}\right) \\
s_{\{k\} q-}^{k_{1}} & =\sqrt{2}\left\langle C_{k_{1} q}(\theta, 0) C_{k q}\left(\theta_{j^{\prime}}, 0\right) \sin \left(q \phi_{j^{\prime}}\right)\right\rangle \\
& =\sqrt{2} \frac{1}{N_{j^{\prime}}} \sum_{i=1}^{N_{j^{\prime}}} C_{k_{1} q}\left(\theta^{(i)}, 0\right) C_{k q}\left(\theta_{j^{\prime}}^{(i)}, 0\right) \sin \left(q \phi_{j^{\prime}}^{(i)}\right) \\
s_{\{k\} 0}^{k_{1}} & \left.=\left\langle P_{k_{1}}(\cos \theta) P_{k}\left(\cos \theta_{j^{\prime}}\right)\right)\right\rangle \\
& =\frac{1}{N_{j^{\prime}}} \sum_{i=1}^{N_{j^{\prime}}} P_{k_{1}}\left(\cos \theta^{(i)}\right) P_{k}\left(\cos \theta_{j^{\prime}}^{(i)}\right)
\end{aligned}
$$

where $\theta^{(i)}, \theta_{j^{\prime}}^{(i)}$ and $\phi_{j^{\prime}}^{(i)}$ are, respectively, the scattering angle and the polar angles of $\boldsymbol{j}^{\prime}$ for the $i$ th trajectory and $N_{j^{\prime}}$ is the number of trajectories ending in a specific rotational state of the NO molecule. In particular, the DCS, corresponding to $k=0$, $q=0$, is calculated as series in Legendre polynomials whose coefficients are the $S_{\{0\} 0}^{k 1}$ of the last equation.

The nonvanishing real polarization parameters are calculated as

$$
\begin{gathered}
a_{q+}^{\{k\}}=(-1)^{q} \sqrt{2}\left\langle C_{k|q|}\left(\theta_{j^{\prime}}, 0\right) \cos \left(q \phi_{j^{\prime}}\right)\right\rangle, \quad k=\text { even } \\
a_{q-}^{\{k\}}=(-1)^{q} \sqrt{2}\left\langle C_{k|q|}\left(\theta_{j^{\prime}}, 0\right) \sin \left(q \phi_{j^{\prime}}\right)\right\rangle, \quad k=\text { odd } \\
a_{0}^{\{k\}}=\left\langle P_{k}\left(\cos \theta_{j^{\prime}}\right)\right\rangle, k=\text { even }
\end{gathered}
$$

Finally, the two vector-correlation $\left(\boldsymbol{k}-\boldsymbol{j}^{\prime}\right.$ and $\left.\boldsymbol{l}-\boldsymbol{j}^{\prime}\right)$ can be obtained as an expansion in Legendre polynomials characterized by the angles $\theta_{j^{\prime}}$ and $\theta_{l j^{\prime}}$ respectively. ${ }^{5,13}$ In particular, the $P\left(\cos \theta_{j^{\prime}}\right)$ distribution can be written as:

$$
P\left(\cos \theta_{j^{\prime}}\right)=\frac{1}{2} \sum_{k=0}^{\infty} a_{0}^{\{k\}} P_{k}\left(\theta_{j^{\prime}}\right), \quad k=\text { even }
$$

and a similar expression for the $\boldsymbol{l} \boldsymbol{j}^{\prime}$ correlation in terms of $\cos \theta_{l j^{\prime}}$ that contains, however, even and odd terms.
The dihedral angle distribution of the $\boldsymbol{k}-\boldsymbol{k}^{\prime}-\boldsymbol{j}^{\prime}$ and $\boldsymbol{k}-\boldsymbol{l}-\boldsymbol{k}^{\prime}$ three-vector correlation, $P\left(\phi_{j^{\prime}}\right)$ and $P\left(\phi_{l}\right)$, for inelastic trajectories are calculated by expansion in a Fourier series. ${ }^{5,9}$ The $P\left(\phi_{j^{\prime}}\right)$ is given by

$$
P\left(\phi_{j^{\prime}}\right)=\frac{1}{2 \pi}\left[1+\sum_{n \text { even } \geq 2}^{\infty} a_{n} \cos n \phi_{j^{\prime}}+\sum_{n \text { odd } \geq 1}^{\infty} b_{n} \sin n \phi_{j^{\prime}}\right]
$$

The coefficients $a_{n}$ and $b_{n}$ of this Fourier expansion can be written in terms of the real polarization parameters, $a_{q \pm}^{\{k\}}$, and can be calculated as

$$
\begin{aligned}
& a_{n}=2\left\langle\cos n \phi_{j^{\prime}}\right\rangle \\
& b_{n}=2\left\langle\sin n \phi_{j^{\prime}}\right\rangle
\end{aligned}
$$

In all these equations, the brackets indicate the averaging over all trajectories leading to a specified rotational final state.

It must be remarked that eqns. (2), (3), (5), (17) and (18) correspond to the purely classical description of the polarization of the rotational angular momentum in terms of the classical angles $\theta_{j^{\prime}}$ and $\phi_{j^{\prime}}$, which define the direction of $\boldsymbol{j}^{\prime}$. Very recently, however, a theoretical treatment for the quantal spatial distribution of angular momenta has been developed in terms of states of minimum uncertainty, which allows to define population distribution functions ${ }^{41}$ and overcomes the limitations of the widely used vector model. It emerges from this treatment that, apart from dynamical effects, classical and quantum mechanical polarization moments can be directly compared, but the prescription for a unified description and representation of the angular momentum distributions implies the introduction of the Clebsch-Gordan factor $\langle j j, k 0 \mid j j\rangle$. Since the present calculations are purely classical, we will not use this prescription in this work in spite of the limitations resulting from the adoption of the vector model.

\section{Results and discussion}

The only rotational angular momentum relevant for the experiments under consideration ${ }^{30,31}$ is that of the collision products, $j^{\prime}$, since the NO molecules are initially in their ground rotational state and implicitly the NO is considered as a closed shell molecule.

A summary of the lower orientation and alignment polarization moments $(k \leq 2)$ in the $\boldsymbol{k}-\boldsymbol{k}^{\prime}$ frame, averaged on the scattering angle, $\theta$, is represented in Fig. 2 as a function of $j^{\prime}$.

The $a_{1-}^{\{1\}}$ parameter contains information about the orientation of $\boldsymbol{j}^{\prime}$ along the $y$-axis (orientation moments correspond to $k$ odd, whereas alignment moments are those with $k$ even). Its value is represented as a function of $j^{\prime}$ in the upper panel of this figure, and stays relatively close to zero for all the $j^{\prime}$ values, showing that there is not a strong orientation of $\boldsymbol{j}^{\prime}$ along the $y$-axis. For the lowest $j^{\prime}$ values this parameter is slightly positive indicating a certain preference for orientation parallel to $y$ (i.e. along the positive $y$-axis); for $j^{\prime}>6$ the tendency is inverted, and for $j^{\prime}>14$ reaches a negative value, which is still far from the limiting value of -1 for this moment. The parameter $a_{0}^{2}$, which represents the alignment with respect the initial relative velocity, is initially positive, then zero for $j^{\prime}=$ 3-6 and finally tends to its lowest (i.e. most negative) limit with increasing $j^{\prime}$. Therefore, for the highest rotational levels, $\boldsymbol{j}^{\prime}$ has a marked propensity to be aligned perpendicular to $z=\boldsymbol{k}$. The $a_{1+}^{\{2\}}$ parameter contains information on the preference for $\boldsymbol{j}^{\prime}$ to be aligned along the $(x+z)$-axis (positive values) or along the $(x-z)$-axis (negative values), that is the tilting of $\boldsymbol{j}^{\prime}$ with respect to $x y$ and $y z$ planes. Its value is positive for all $j^{\prime}$, thus indicating a certain tendency of $\boldsymbol{j}^{\prime}$ to be aligned parallel with respect to $x+z$. Finally, the $a_{2+}^{\{2\}}$ polarization parameter gauges the relative preference for $\boldsymbol{j}^{\prime}$ alignment along the $x$-axis with respect to the $y$-axis. Its value is always negative from $\approx-0.4$ 

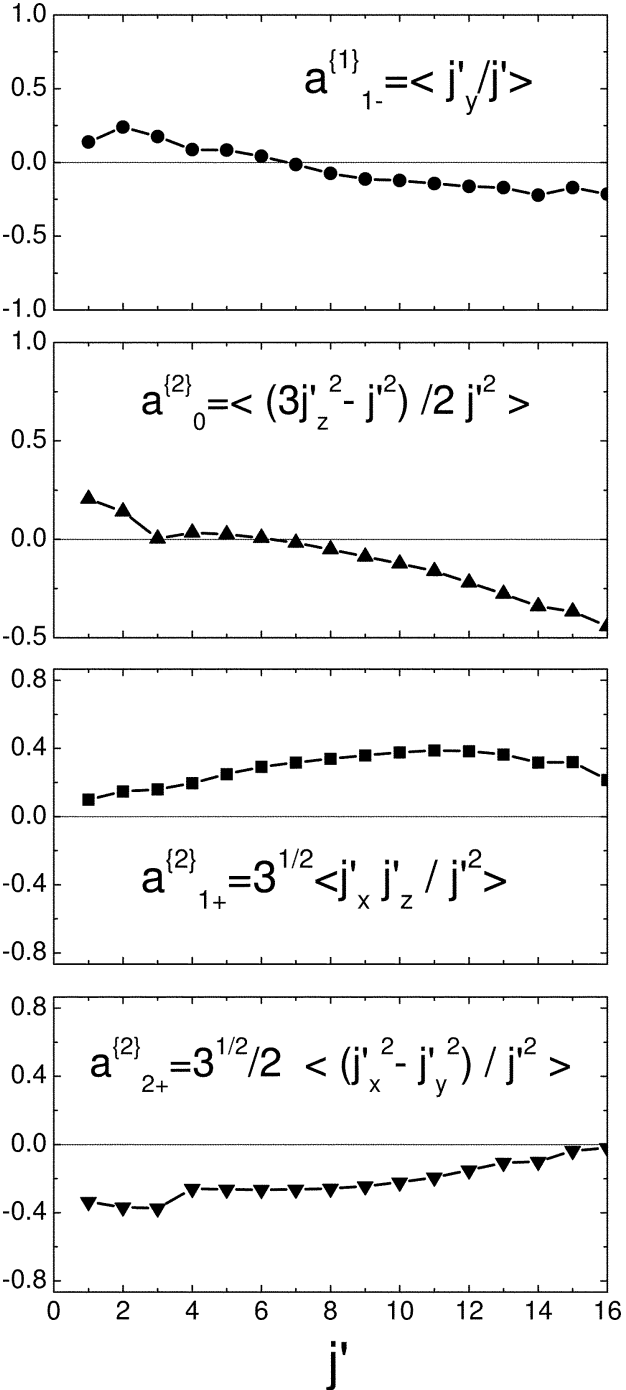

Fig. 2 First four real polarization parameters $a_{q}^{\{k\}}, 11-, 20,21+$ and $22+\left(\right.$ see text) for $\mathrm{Ar}+\mathrm{NO}(j=0) \rightarrow \mathrm{Ar}+\mathrm{NO}\left(j^{\prime}\right)$ as a function of the $\boldsymbol{j}^{\prime}$ final states. The definitions of these polarization parameters in terms of the components of $j^{\prime}$ are also indicated in the figure. The ranges of the vertical axes coincide with limits of the classically allowed values of the corresponding polarization parameters.

to zero with increasing $j^{\prime}$, indicating a decreasing tendency towards alignment of $\boldsymbol{j}^{\prime}$ along the $y$-axis. The classical and quantum mechanical definitions of these polarization moments and their limiting values are given in Tables I and II of ref. 13. For subsequent reference, it must be noticed that the definitions and limiting values differ by a constant factor from those of in ref. 31. Given the definitions adopted in the latter reference, their classical limits for $a_{0}^{\{2\}}$ are $[-1,2]$ and those for $a_{2+}^{\{2\}}$ are \pm 1 . In the present work, as well as in ref. 13 the corresponding limits are $[-1 / 2,1]$ and $\pm \sqrt{ } 3 / 2$, for the 20 and $22+$ moments, respectively. In addition, it should be remarked that the actual limiting values of these polarization parameters in the quantum (experimental) case depend on the value of $j^{\prime}$.

A graphical summary of the polarization behaviour commented on in the previous paragraphs can be found in Fig. 3, where the classical distributions of the direction of $\boldsymbol{j}^{\prime}$ are represented for chosen values of $j^{\prime}$ as polar plots. To calculate these distributions the polarization moments up to $k=2 j$ (the maximum value allowed quantum mechanically) have been used (see ref. 41). This figure shows a distinct evolution in the distribution of $\boldsymbol{j}^{\prime}$ with growing rotational quantum number. For $j^{\prime}=2$, the vector $\boldsymbol{j}^{\prime}$ is predominantly contained on the $y z$ plane with a higher probability to point towards the $+y$-axis. For the $j^{\prime}=6$ and $j^{\prime}=10$ intermediate levels considered, $\boldsymbol{j}^{\prime}$
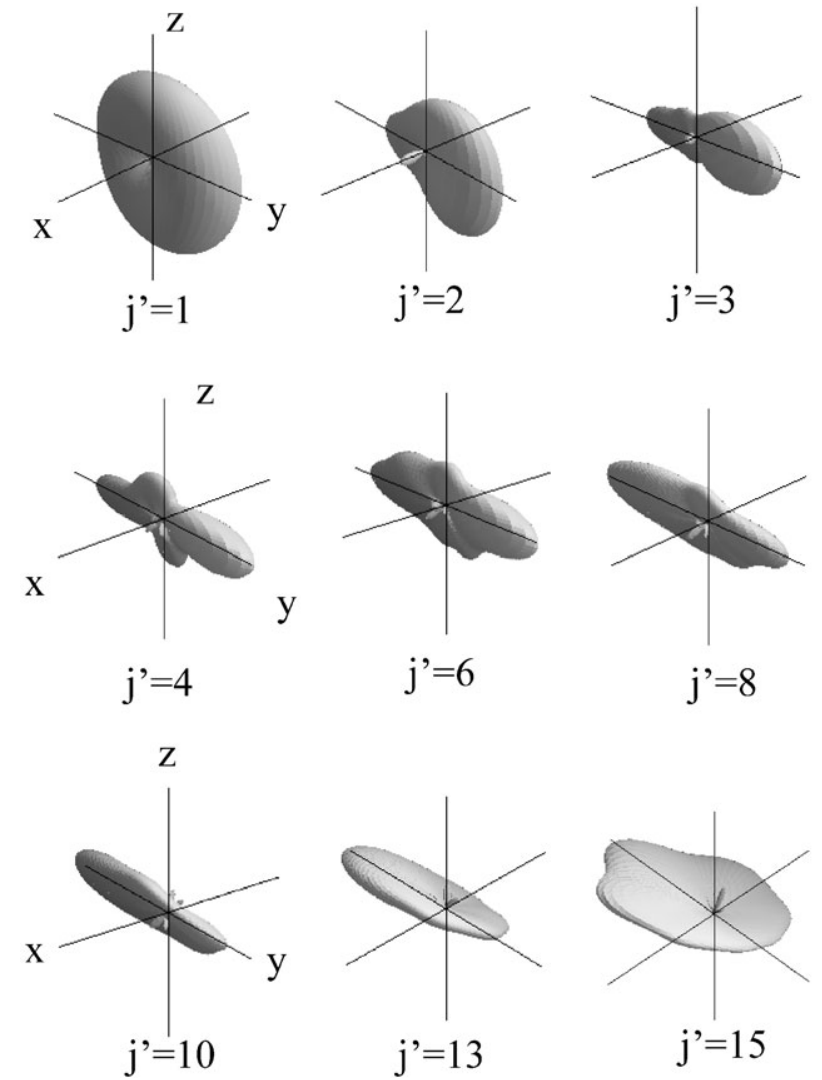

Fig. 3 Polar plots of the distributions of the $\boldsymbol{j}^{\prime}$ vector in the scattering frame ( $\boldsymbol{k}$ along the $z$-axis and $y$-axis parallel to $\boldsymbol{k} \times \boldsymbol{k}^{\prime}$ ) for several final $\boldsymbol{j}^{\prime}$ states of the NO molecule. These plots represent the polarization of $j^{\prime}$ averaged over scattering angles.

becomes more strongly polarized along the $y$ axis and strongly tilted along the $(x+z)$. As $j^{\prime}$ grows even further the distributions become increasingly flatter lying on a plane which is still tilted with respect to the $x y$-plane, and have some preference for the negative direction of the $y$-axis.

The classical probability distributions of $\cos \theta_{j^{\prime}}$, that is of the cosine of the angle between $j^{\prime}$ and the direction of the initial relative velocity of the $\mathrm{Ar}+\mathrm{NO}$ colliding pair, $\boldsymbol{k}$, are shown in the left panel of Fig. 4 for a series of $j^{\prime}$ values. These distributions contain only terms with $k$ even and $q=0$ (thus symmetric with respect to $\theta_{j^{\prime}}=90^{\circ}$ ). For $j^{\prime}=2$ the $\boldsymbol{j}^{\prime}$ vector can form any angle with $\boldsymbol{k}$ but shows a certain preference for a parallel or antiparallel orientation, with increasing $j^{\prime}$ the distribution becomes more isotropic and for $j^{\prime}>10$ parallel and antiparallel orientations begin to disappear. For $j^{\prime}=15$, which is close to the limit energetically allowed, the angle between $\boldsymbol{k}$ and $\boldsymbol{j}^{\prime}$ is constrained to the $60^{\circ}-150^{\circ}$ angular interval and nearly isotropically distributed within this range.

The right panel of the same figure displays the corresponding dihedral angle classical distributions $P\left(\phi_{j^{\prime}}\right)$ of $\boldsymbol{j}^{\prime}$ in the $\boldsymbol{k}-\boldsymbol{k}^{\prime}$ frame (see Fig. 1). In contrast with the $P\left(\cos \theta_{j^{\prime}}\right)$, the $P\left(\phi_{j^{\prime}}\right)$ distribution contains information about $k$-odd moments as shown in section II. The marked dependence of this distribution on the final rotational state of NO is manifest in this figure. For the lowest excited state shown, $j^{\prime}=2$, the distribution has a pronounced narrow peak centered at $\phi_{j^{\prime}}=90^{\circ}$, and a smaller and broader maximum at $\phi_{j^{\prime}}=270^{\circ}$ (i.e. $j^{\prime}$ is preferentially oriented along the positive $y$ axis of Fig. 1). This asymmetry in the $P\left(\phi_{j^{\prime}}\right)$ distribution, reflects a preferential orientation of $j^{\prime}$ and implies that the majority of NO molecules excited to $j^{\prime}=2$ in the course of the collision undergo counterclockwise rotation with respect to the $\boldsymbol{k}-\boldsymbol{k}^{\prime}$ plane. With increasing $j^{\prime}$, the maximum around $270^{\circ}$ becomes gradually more important, reflecting a growing amount of NO excited 

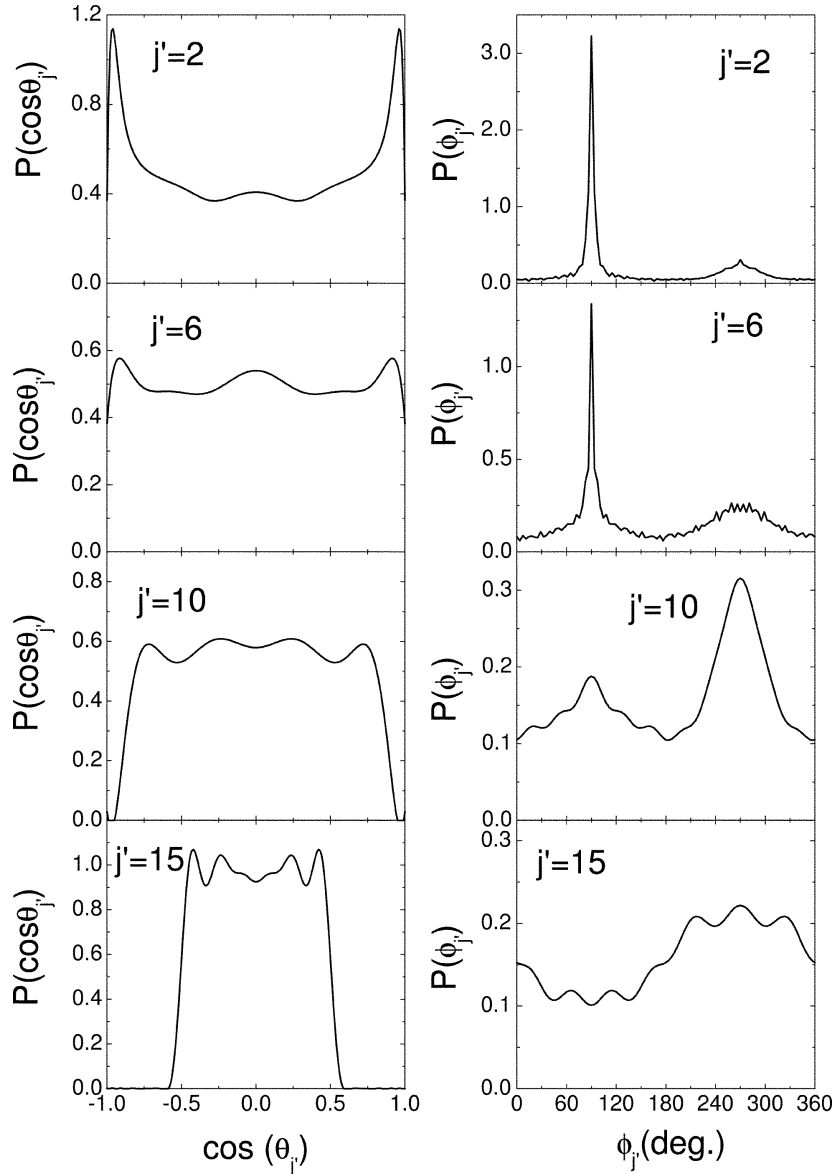

Fig. 4 Left: Probability distribution $P\left(\cos \theta_{j^{\prime}}\right)$, as a function of $\cos$ $\left(\theta_{j^{\prime}}\right)$ for some specific values of $j^{\prime}$. This distribution contains information about the alignment of $\boldsymbol{j}^{\prime}$ with respect to $\boldsymbol{k}$, and thus it is symmetric about $\theta j^{\prime}=90^{\circ}$. Right: Distribution of the dihedral angle $\phi_{j^{\prime}}$ between the $\left(\boldsymbol{k}-\boldsymbol{k}^{\prime}\right)$ - and the $\left(\boldsymbol{k}-\boldsymbol{j}^{\prime}\right)$-planes. This distribution contains information about the orientation of $\boldsymbol{j}^{\prime}$ with respect to the $\left(\boldsymbol{k} \times \boldsymbol{k}^{\prime}\right)$-axis.

molecules rotating also clockwise with respect to the scattering plane, and the peak at $90^{\circ}$ gets broader. For the highest rotational level considered, $j^{\prime}=15$, the $P\left(\phi_{j^{\prime}}\right)$ distribution has a large isotropic character with a broad maximum around $270^{\circ}$ and a shallow minimum around $90^{\circ}$.

The correlation between the initial orbital angular momentum vector $\boldsymbol{l}$ and the rotational angular momentum of the outgoing NO molecule provides a most interesting clue about the reaction mechanism. The left column of Fig. 5 shows the distribution of angles between $\boldsymbol{l}$ and the rotational angular momentum of NO after the collision. In all the cases shown, both vectors are predominantly parallel, but for the three lower values considered there is also a significant amount of antiparallel orientation. For $j^{\prime}=15$ the antiparallel orientation is practically lost. The right part of this figure displays the distribution of the dihedral angle $\phi_{l}$ between the $\left(\boldsymbol{k}-\boldsymbol{k}^{\prime}\right)$ - and $(\boldsymbol{k}-\boldsymbol{l})$-planes. For $j^{\prime}=2$ the distribution has two parts, a peak with a double maximum centered around $90^{\circ}$ and a less pronounced single peak at $270^{\circ}$; both features are comparatively narrow indicating that the $\boldsymbol{l}$-vector tends to be perpendicular to the $\boldsymbol{k}-\boldsymbol{k}^{\prime}$ scattering plane. As can be seen in the bottom panel of Fig. 1, the maximum at $90^{\circ}$ corresponds to a deflection caused by attractive interactions (negative deflection angles), whereas that at $270^{\circ}$ is characteristic of a rebound repulsive mechanism (positive deflection angles). For $j^{\prime}>3$ values, the maximum around $90^{\circ}$ disappears indicating that the excitations of NO to these levels are just due to repulsive interactions, as discussed in ref. 34. In addition the distribution becomes wider and splits on top showing two peaks at the edges. The gradual broadening of the distribution indicates
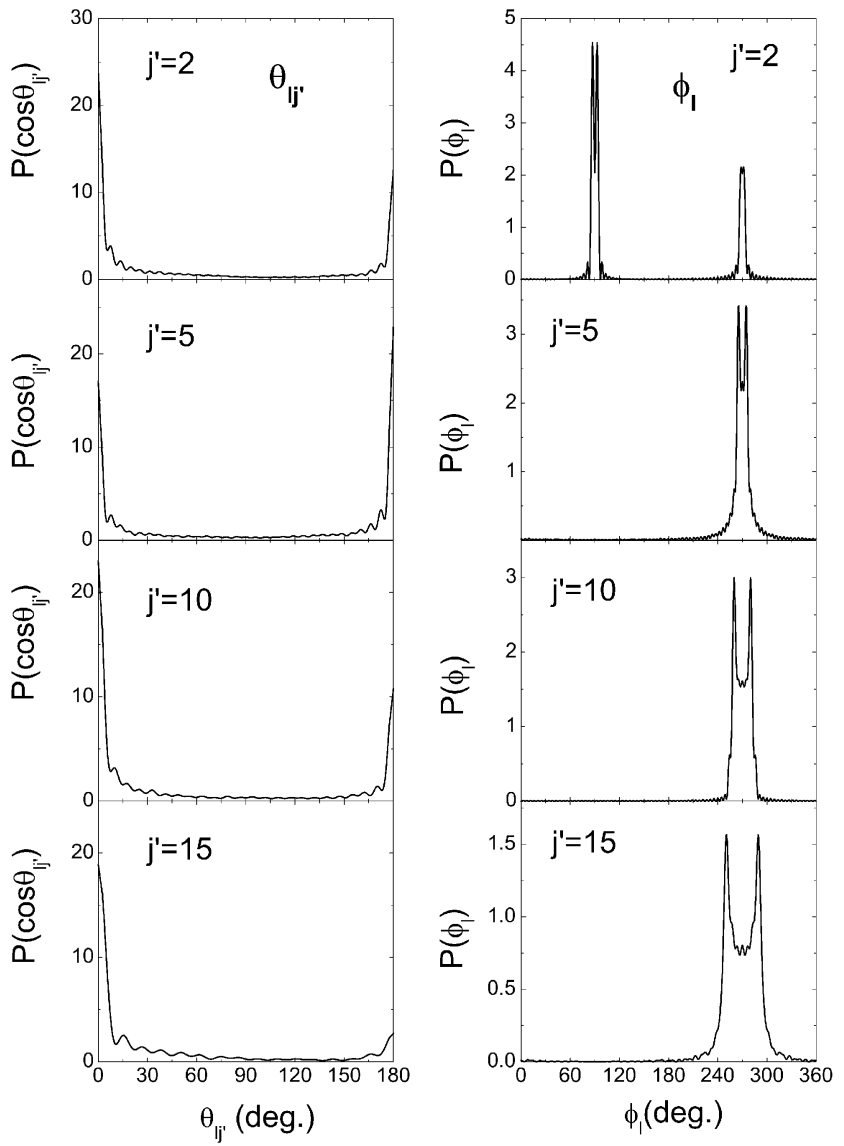

Fig. 5 Left: Probability distributions of the cosine of the angle between the initial orbital angular momentum $\boldsymbol{l}$ and the rotational angular momentum of the NO product, as a function of the angle between both vectors $\theta_{l j^{\prime}}$ for the same $j^{\prime}$ states shown in Fig. 4. In the four cases, the vectors are predominantly parallel, although for the three lower values of $j^{\prime}$ there is still an important contribution of antiparallel configurations. For $j^{\prime}=15$, both vectors are mostly parallel. Right: Distribution of the dihedral angle $\phi_{l}$ between the $\left(\boldsymbol{k}-\boldsymbol{k}^{\prime}\right)$ - and the $(\boldsymbol{k}-\boldsymbol{l})$-planes. For $j^{\prime}=2$ the distribution shows two contributions, one around $90^{\circ}$, mainly due to attractive contributions, and the second peak, centered at $270^{\circ}$, that increases for large values of $j^{\prime}$, that corresponds to the repulsive interactions. The small width of both peaks, indicates that the $\boldsymbol{l}$ vector tends to be perpendicular to the $\left(\boldsymbol{k}-\boldsymbol{k}^{\prime}\right)$-plane.

that the tendency of $\boldsymbol{l}$ to be perpendicular to the $\left(\boldsymbol{k}-\boldsymbol{k}^{\prime}\right)$-plane decreases with growing $j^{\prime}$. In the first case, the trajectory, which can be either attractive $\left(\phi_{l} \approx 90^{\circ}\right)$ or repulsive $\left(\phi_{l} \approx 270^{\circ}\right)$, is coplanar i.e. the scattering plane is perpendicular to the initial (orbital) angular momentum vector $\boldsymbol{l}$, as illustrated in the lower part of the Fig. 1, where characteristic trajectories are shown. In the second case the coplanarity is lost and the scattering plane is no more perpendicular to $\boldsymbol{l}$.

Renormalized polarization dependent differential cross sections, PDDCS, ${ }^{13}$ for selected values of the final rotational quantum number are shown in Figs. 6 and 7. The classical $j^{\prime}$ values chosen correspond to the NO rotational quantum numbers presented in the work of Lorenz et al. ${ }^{30}$ For each rotational level, the $k=0, q=0 \mathrm{PDDCS}$ is proportional to the usual differential cross section (see section II). These classical DCSs and their evolution with the rotational quantum number, including the appearance of a forbidden scattering range toward lower angles with increasing $j^{\prime}$, were already commented on in a previous publication. ${ }^{34}$ The rest of the PDDCSs displayed contain in principle the stereodynamical information needed for comparison with the measurements of refs. 30 and 31 and their individual physical meaning is the same as that of the corresponding polarization parameters already shown in Fig. 2, but resolved in scattering angles. The $\rho_{0+}^{\{2\}}$ and $\rho_{2+}^{\{2\}}$ 

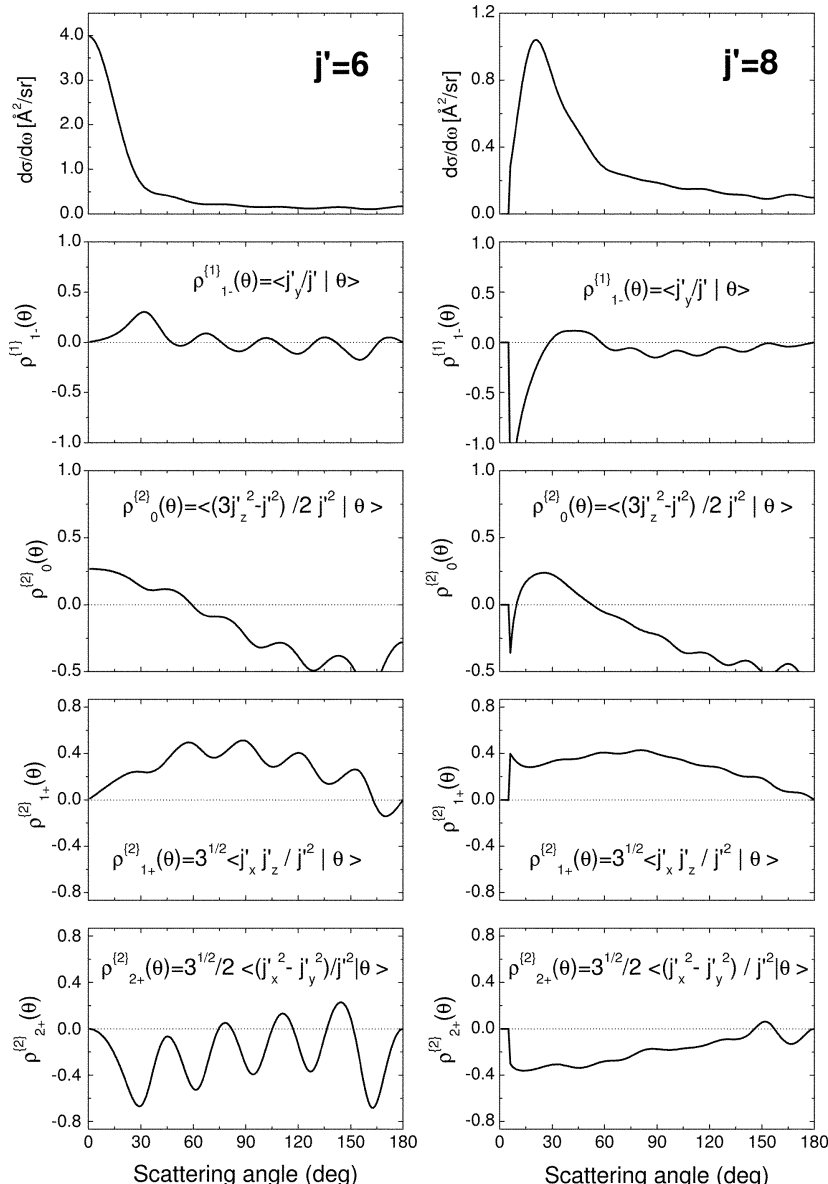

Fig. 6 Polarization-dependent differential cross-section (PDDCSs) for $j^{\prime}=6$ (left column) and for $j^{\prime}=8$ (right column). Top panels in both columns are the differential cross sections.

PDDCSs for $j^{\prime}=8$ and $j^{\prime}=15$ can be directly compared to the $A_{0+}^{\{2\} \mathrm{COL}}$ and $A_{2+}^{\{2\} \mathrm{COL}}$ of ref. 31 (see Figs. 3 and 4 of this reference) just by taking into account the constant conversion factor mentioned above. The agreement between the present values and the calculations of ref. 31 is good in the classically allowed angular range. In both cases, for $j^{\prime}=8$ no appreciable alignment is found for the lower angles, but the rotational angular momentum shows an increasing propensity to be aligned perpendicular to $\boldsymbol{k}$ with growing scattering angle. The measurements give the same qualitative trend, but the degree of experimental alignment is smaller for the largest angles. For $j^{\prime}=15$ the calculations predict a more marked tendency for a perpendicular alignment between $\boldsymbol{j}^{\prime}$ and $\boldsymbol{k}$ at low angles which is again increased with growing scattering angle. The experimental alignment is indeed already appreciable for the lower angles, but it is always smaller than that from the theoretical predictions and shows no definite trend over the whole angular range.

With the data from Figs. 6 and 7 one can easily construct the quantity $Q_{j^{\prime}}(\theta)$ :

$$
Q_{j^{\prime}}(\theta)=\frac{6 h_{\mathrm{j}^{\prime}}^{(1)} \rho_{1-}^{\{1\}}\left(\theta, j^{\prime}\right)}{4+h_{\mathrm{j}^{\prime}}^{(2)}\left[\left(\rho_{0+}^{\{2\}}, j^{\prime}\right)+3 \rho_{2+}^{\{2\}}\left(\theta, j^{\prime}\right)\right]},
$$

with

$$
\begin{gathered}
h_{j^{\prime}}^{(1)}=-\left(j^{\prime}+1\right) \div\left[j^{\prime}\left(j^{\prime}+1\right)\right]^{1 / 2} \\
h_{j^{\prime}}^{(2)}=-j^{\prime} /\left(2 j^{\prime}+3\right)
\end{gathered}
$$

for R-branch transitions. ${ }^{42}$ The essential orientational information provided by $Q_{j^{\prime}}(\theta)$ is contained in the $\rho_{1-}^{\{1\}}\left(\theta, j^{\prime}\right)$ polarization moment, which takes different signs for the
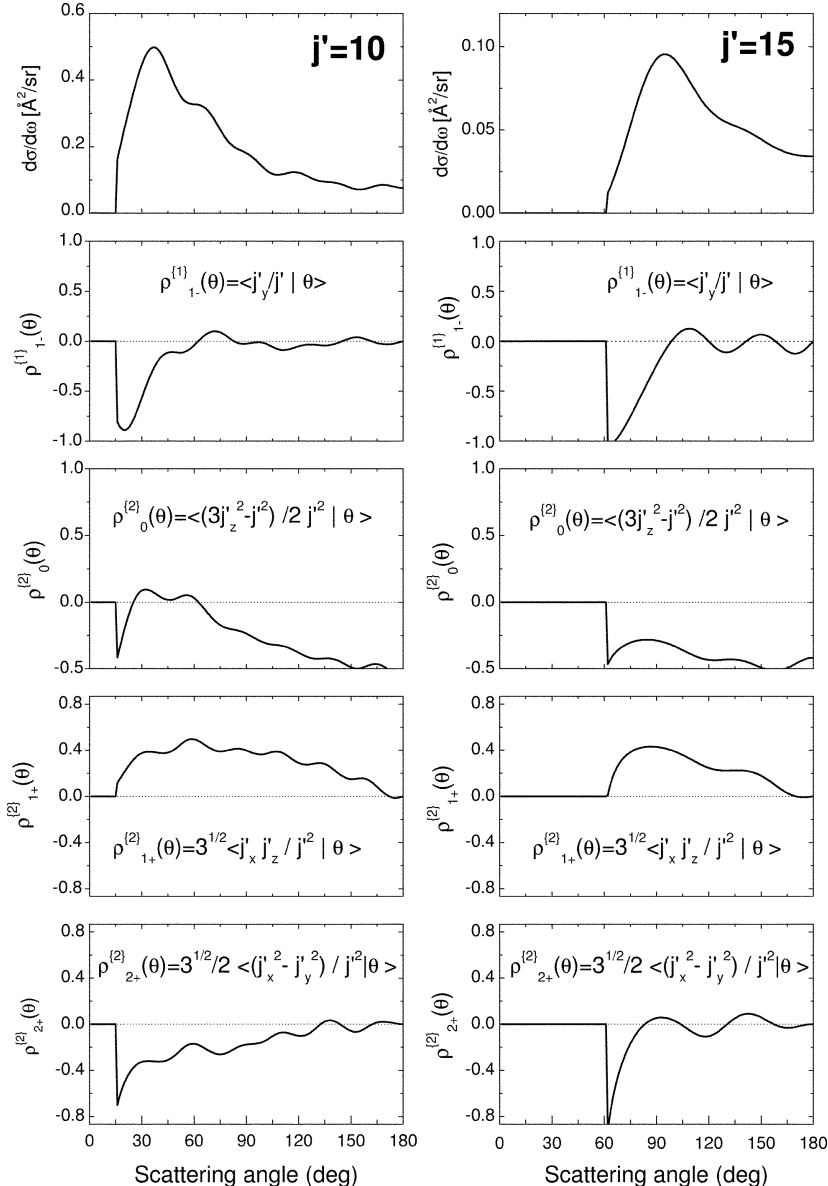

Fig. 7 Same as Fig. $6 j^{\prime}=10$ (left column) and $j^{\prime}=15$ (right column).

two possible senses of rotation of the NO molecule after the collision. Note that the sign of $Q_{j^{\prime}}(\theta)$ is opposite to that of the corresponding $\rho_{1-}^{\{1\}}\left(\theta, j^{\prime}\right)$ due to the $h_{j^{\prime}}^{(1)}$ factor appearing in eqn. (21).

Fig. 8 compares the classical $Q_{j^{\prime}}(\theta)$ with the theoretical and experimental $Q_{j^{\prime}}(\theta)$ reported by Lorenz et al. ${ }^{30}$ The first lobe in the QCT $Q_{j^{\prime}}(\theta)$ is negative for $j^{\prime}=6$ and positive for the rest of the rotational states considered. For the three largest $j^{\prime}$, the angular range of this first lobe extends to larger angles with increasing $j^{\prime}$. This behaviour is in qualitative agreement with the experimental and QM results. However, the QCT $Q_{j^{\prime}}(\theta)$ differ from the quantal and experimental $Q_{j^{\prime}}(\theta)$ in some important aspects: they exhibit a neat angular threshold followed by a sharp, too intense and narrow maximum and the oscillatory pattern observed, for $j^{\prime}<15$ is less marked and shifted in angles. The sharp increase observed in the QCT calculations is due to the fact that the normalization of the various $\rho_{q \pm}{ }^{\{k\}}\left(\theta, j^{\prime}\right)$ implies the division by $d \sigma_{00} / d \omega$ (see eqn. (6)), which is very close to zero immediately after the classical angular threshold.

Fig. 8 also includes the results of a simple classical model based on hard sphere-rigid ellipsoid collisions, reported also by Lorenz et al. ${ }^{30}$ As expected for a classical model, a forbidden angular range increasing with growing $j^{\prime}$ is also found here although the sharp post-threshold rise like the one described in the previous paragraph, has not been represented. This impulsive model predicts an appreciable rotational orientation (i.e. $Q_{j^{\prime}}(\theta) \neq 0$ ) for certain scattering angles, but $Q_{j^{\prime}}(\theta)$ only shows a small oscillation around zero in the angular range $<90^{\circ}$ for $j^{\prime}=8$ and 10 , in contrast with the QM results and with the experimental observations. The authors advance two possible explanations for this deficiency: the neglect of the attractive part of the potential in the simple model based on rigid bodies, or the inability of the classical approach to account for interference effects. The present QCT $Q_{j^{\prime}}(\theta)$, 
$Q_{\exp }(\theta)$
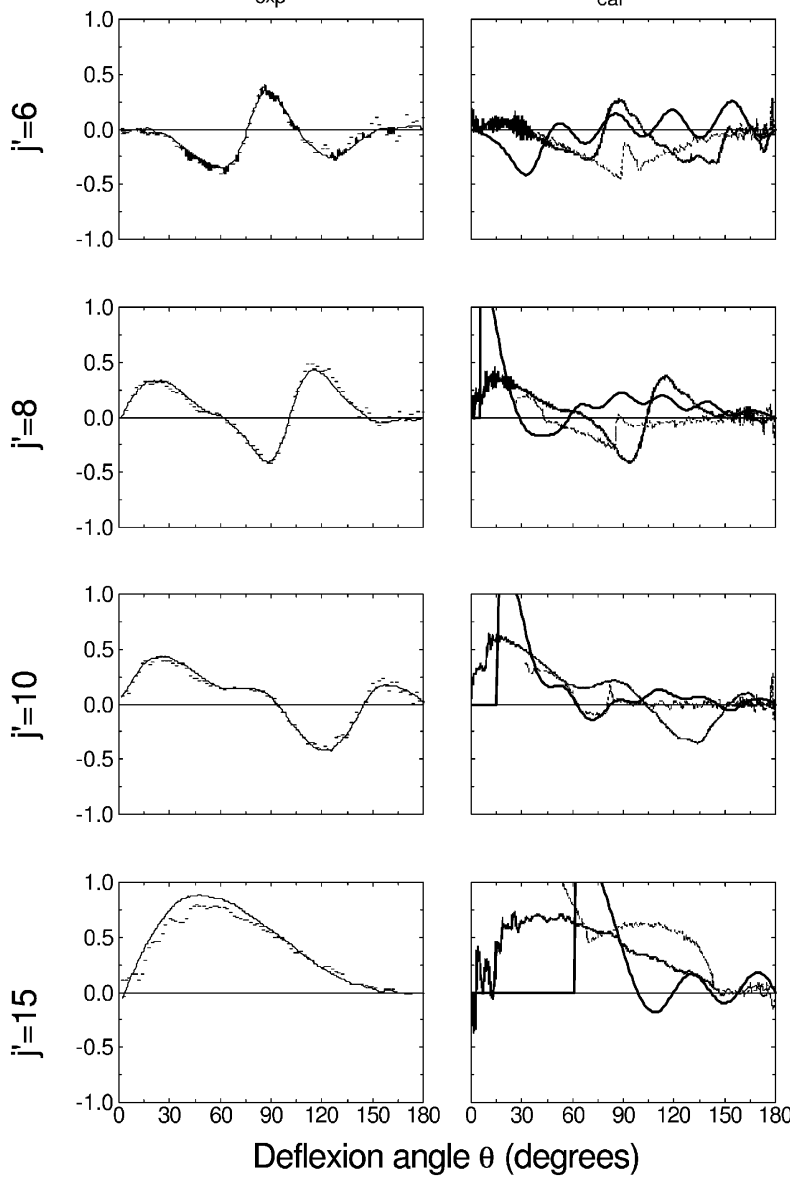

Fig. 8 Comparison of the $Q_{\exp }(\theta)$ factor (left panels) and the $Q_{\text {cal }}(\theta)$ (right panels) for the specified $j^{\prime}$ values. In the results shown in the right column, the solid thick line are the present results, while the QM results (thick dotted line), and the classical calculation of the ellipsoid model (narrow line) are taken from ref. 30. Also the experimental data, in the left column, is taken from this reference.

derived from calculations carried out on an accurate PES, show some large-angle oscillations between positive and negative values for the lowest $j^{\prime}$, and suggest that the consideration of the attractive part of the potential can give an additional mechanism allowing for a different sense of rotation in the excitation of a given state of NO. However, the amplitude of these oscillations is small and they do not appear at the same angles as those from experiment and from QM calculations. The classical description, even with the consideration of an accurate potential including attraction, is thus insufficient to account for the observations. Most probably QM interference effects are responsible for the observed oscillatory pattern as suggested by Lorentz et al. ${ }^{30}$ and by Althorpe. ${ }^{43}$

An additional difference between the classical ellipsoid model of refs. 30,31 and the present QCT calculations consists in the dimensions of the ellipsoid used in these references. The authors quote values of 3.63 and $2.82 \AA$ for the major and minor axis respectively. These values were fitted to obtain a good agreement with the position of the rotational rainbows in the differential cross section. In the present work the full $a b$ initio $V_{\text {sum }}$ potential of ref. 32 is used without any adjustment. Actually, the values of the axes obtained with this potential for the zero energy contour, that is for the purely repulsive potential, are 3.75 and $3.2 \AA$. The different dimensions of the two ellipsoids are most probably responsible for the differences found between the present calculations and the classical results from refs. 30,31 for the scattering into the excited NO levels where attractive interactions are negligible.
In the experiment and in all the calculations the largest orientation and alignment with respect to $\boldsymbol{k}$ is obtained for the highest rotational excitation $\left(j^{\prime}=15\right)$. This result can be rationalized by taking into account that this high excitation regime, dominated by repulsive interactions, ${ }^{34}$ leads to a near complete transfer of the collision energy into molecular rotation. Under these conditions, that necessarily imply a small recoil velocity, a subsequent collision ("chattering") may take place without perceptible motion of the slow moving atom. ${ }^{44,45}$ The net effect may be a considerable reduction in the rotational energy of the outgoing molecule. In order to maintain the high rotational excitation, the atom must hit the molecule close to one of its ends and the molecular axis must rotate away from the deflected atom. If the molecular axis rotates toward the slowly moving atom a "second collision" leading to de-excitation will take place. ${ }^{44}$ This condition imposes the observed correlation between the sense of rotation and the $\left(\boldsymbol{k}-\boldsymbol{k}^{\prime}\right)$-plane.

Chattering collisions are frequent in the $\mathrm{Ar}-\mathrm{NO}$ system under consideration, and can be easily visualized in the present QCT calculations. In Fig. 9, two trajectories leading initially to NO $\left(j^{\prime}=15\right)$ are compared; in the first of them (upper part) the excitation is maintained while the collision partners fly apart. In the second (lower part), the molecule becomes partially deexcited after experiencing a second collision with the slow moving atom. Note that in the course of the first trajectory, the ArO distance, $R_{\mathrm{ArO}}$, reaches an appreciably smaller value than the $\mathrm{ArN}$ distance, $R_{\mathrm{ArN}}$, and that the center of mass distance, $R_{\mathrm{Ar}-\mathrm{NO}}$, increases markedly immediately after the $R_{\mathrm{ArO}}$ minimum as the molecule rotates away from the departing atom. In contrast, when a "second collision" takes place $R_{\mathrm{Ar}-\mathrm{NO}}$ does not vary much during the time between the minima of $R_{\mathrm{ArN}}$ and $R_{\mathrm{ArO}}$, which have approximately the same value. The slower variation of $R_{\mathrm{Ar}-\mathrm{NO}}$ is associated with the chattering process in which the rotating molecule approaches the atom. This increase in the interaction time due to the chattering leads likely to a decrease in the orientation and alignment of the final rotational angular momentum, which is reflected, for instance, in the smaller (absolute) values of the $a_{1-}^{\{1\}}$ and $a_{0}^{\{2\}}$ for $j^{\prime}=10$ as compared with those for $j^{\prime}=$ 15 (see Fig. 3). In any case, the rotational motion leading to the "second collision" is only a fraction of the full rotational period of the molecule. It must be stressed that the definition of a chattering collision adopted in this work is based on: (i) a maximum in the translational to rotational energy transfer followed by a decreases in the rotational excitation of the diatom with respect to this maximum value (ii) neat consecutive interactions with the two ends of the molecule. These two conditions are reflected in the trajectory portrayed in the lower panels of Fig. 9. With the mass ratio of the Ar-NO system, it cannot be expected that the Ar moves away appreciably during the course of the interaction. A chattering collision under these conditions is not necessarily characterized by two well separate collisions in which the potential switches off and on, but rather as a somewhat prolonged collision in which the molecule has some time to rotate. In this sense, chattering is relatively frequent in reactive collisions even for systems in which long lived trajectories can be ruled out completely.

Note that in Fig. 9 the trajectory leading to the highest final excitation corresponds to a relatively large impact parameter, $b$, within the repulsive regime, (but certainly much smaller than the $b$ values larger than $4 \AA$ characteristic of attractive interactions that can lead to the excitation of low $\left.j^{\prime}\right) .{ }^{34}$ This is found to be a general behaviour: relatively large impact parameters and a near perpendicular orientation between the $\boldsymbol{k}$ vector and the internuclear axis of the molecule favor a large transfer of momentum and impart the appropriate sense of rotation allowing the "escape" of the atom before a second collision takes place, in accordance with intuitive expectations. Shorter impact parameters and smaller angles between $\boldsymbol{k}$ and the internuclear axis are required in order to let the molecule 

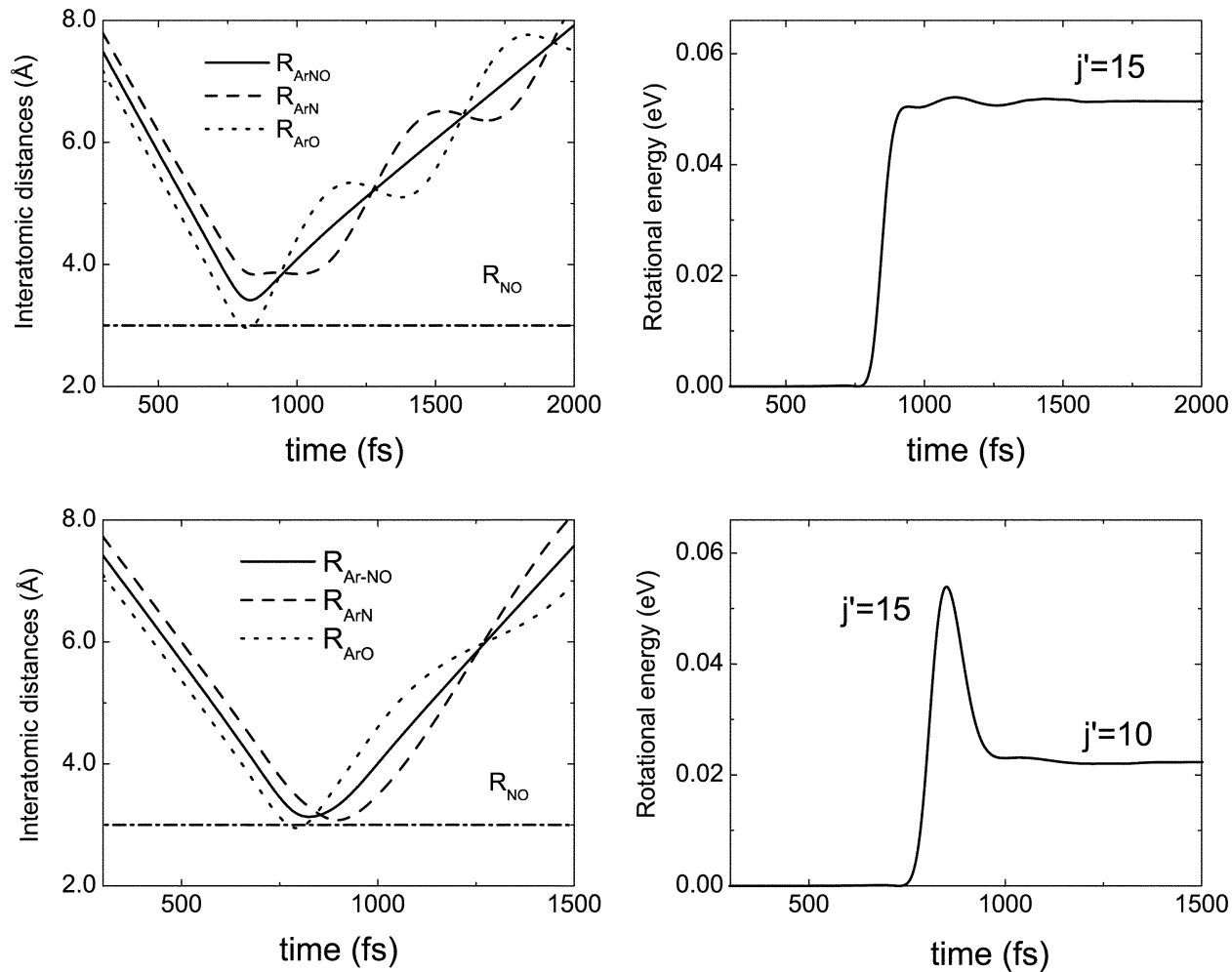

Fig. 9 Left panels: Internuclear distances $R_{\mathrm{ArN}}$ (dashed line), $R_{\mathrm{ArO}}$ (dotted line) and $\mathrm{Ar}-\mathrm{NO}$ center-of-mass distance, $R_{\mathrm{Ar}-\mathrm{NO}}$ (solid line) as a function of time for two selected trajectories. Right panels: Variation of the rotational energy of the NO with time. The trajectory represented in the top panel with impact parameter $b=2.02 \mathrm{~A}$ yields NO in $j^{\prime}=15$. Once the impulsive energy transfer has taken place the rotational energy remains constant. The trajectory of the lower panels, with a small impact parameter $b=0.37 \AA$, is typical of "chattering"; initially the transferred rotational energy reaches a value corresponding to $j^{\prime}=15$. The rotation of the NO causes the Ar atom to interact with the other end of the molecule (see left lower panel) and as a result of this subsequent interaction the rotational excitation decreases to $j^{\prime}=10$.

rotate towards the departing atom and provoke a second interaction with the opposite molecular end before the collision partners fly apart.

Based on the relative decrease of the rotational energy, "chattering collisions" can be quantified more precisely. As an instance, calculations carried out for trajectories ending into $j^{\prime}=8$ show that $\approx 38 \%$ of them have reached a rotational energy slightly larger than that corresponding to $j^{\prime}=9$. The efficiency of a "second collision" to reduce the maximum rotational energy decreases rapidly as $\Delta E_{\text {rot }}$ increases. As an example, only $9 \%$ of the trajectories into $j^{\prime}=8$ arise from trajectories whose maximum rotational energy was that corresponding to $j^{\prime} \geq 13$; that is, with $\Delta E_{\text {rot }} \geq 18.6 \mathrm{meV}$. As commented on above, chattering collisions require sufficiently small impact parameters. The maximum $b$ for trajectories ending in $j^{\prime}=8$ is $3.7 \AA$, however no chattering is found for trajectories with $b>3 \AA$. For chattering from a maximum rotational energy corresponding to $j^{\prime} \geq 13$, the maximum impact parameter is only $1.9 \AA$. In addition, those trajectories experiencing a second encounter are more isotropically distributed in the relevant directions of the angular momenta involved. For example, the directions of the initial orbital angular momentum and the rotational angular momentum of the outgoing molecule are less correlated for chattering collisions. Trajectories leading directly to $j^{\prime}=8$ show an appreciable alignment between the two vectors, i.e. $\boldsymbol{j}^{\prime}$ and $\boldsymbol{l}$ are predominantly parallel and to a lesser extent antiparallel. In chattering trajectories, parallel and antiparallel orientations between these two vectors have the same propensity, and moreover the distribution is considerably more isotropic.

\section{Summary and conclusions}

The present QCT results predict a distinct evolution in the stereodynamical properties of the $\mathrm{Ar}+\mathrm{NO}(j=0)$ rotational excitation at $66 \mathrm{meV}$ with increasing final rotational state of the NO molecule. In particular, a strong orientation of the final rotational angular momentum $\boldsymbol{j}^{\prime}$ with respect to the scattering $\boldsymbol{k}-\boldsymbol{k}^{\prime}$ plane is found for the highest accessible $j^{\prime}$ levels in qualitative agreement with experimental and quantum mechanical data. Secondary collisions of the fast rotating molecules with the slow moving atom are found in the calculations and can induce a depletion of the highest excited rotational states of $\mathrm{NO}$, selective to the sense of rotation. ${ }^{44}$

For the excitation of the lower $j^{\prime}$ values, the QCT calculations, that involve the attractive part of the Ar-NO potential, allow for two mechanisms (attractive and repulsive) of rotational excitation that lead to preferential sense of rotation over a given angular range. However the observed oscillations in the sense of rotation are less marked than those from quantum mechanics and experiment and not entirely coincident in angle. Additional effects, most likely interferences as pointed out by Lorenz et $a l .{ }^{30}$ and by Althorpe, ${ }^{43}$ are needed to account for the actual oscillatory pattern observed in the experiments.

\section{Acknowledgements}

This work has been funded by the Ministry of Science and Technology of Spain under grants BQU2002-04627-C02 and REN 2000-1557 and by the European Union through the RTN Reaction Dynamics(HPRN-CT-1999-0007). The research was performed within the Unidad Asociada "Química Física Molecular" between the Universidad Complutense and the CSIC.

\section{References}

1 D. E. Case and D. R. Herschbach, Mol. Phys., 1975, 30, 1537.

2 D. E. Case, G. M. McClelland and D. R. Herschbach, Mol. Phys., 1978, 35, 541 . 
3 G. M. McClelland and D. R. Herschbach, J. Phys. Chem., 1979, 83, 1445 .

4 J. D. Banwell, J. G. Loeser and D. R. Herschbach, J. Phys. Chem., 1983, 87, 2781

5 F. J. Aoiz, M. Brouard, P. A. Enríquez and R. Sayós, J. Chem. Soc., 1993, 89, 1427.

6 N. E. Shafer, A. J. Orr-Ewing, W. R. Simpson, H. Xu and R. N. Zare, Chem. Phys. Lett., 1993, 212, 155.

7 N. E. Shafer-Ray, A. J. Orr-Ewing and R. N. Zare, J. Phys. Chem., 1995, 99, 7591.

8 F. J. Aoiz, M. Brouard and P. A. Enríquez, J. Chem. Phys., 1996, 105, 4964.

9 F. J. Aoiz, M. Brouard, V. J. Herrero, V. Sáez Rábanos and K. Stark, Chem. Phys. Lett., 1997, 264, 487.

10 K. S. Bradley and G. C. Schatz, J. Chem. Phys., 1997, 106, 8464

11 M. P. de Miranda and D. C. Clary, J. Chem. Phys., 1997, 106, 4509 .

12 M. P. de Miranda, D. C. Clary, J. F. Castillo and D. E. Manolopoulos, J. Chem. Phys., 1998, 108, 3142.

13 M. P. de Miranda, F. J. Aoiz, L. Banares and V. Sáez Rábanos, J. Chem. Phys., 1999, 111, 5368.

14 A. Mattheus, A. Fischer, G. Ziegler, E. Gottwald and K. Bergmann, Phys. Rev. Lett., 1986, 56, 712.

15 H. Meyer, Chem. Phys. Lett., 1994, 230, 519.

16 H. Meyer, J. Chem. Phys., 1994, 101, 6697.

17 M. Brouard and J. P. Simons, in Chemical Dynamics and Kinetics of Small Radicals, ed. A. Wagner and K. Liu, World Scientific, Singapore, 1995.

18 H. Meyer, J. Chem. Phys., 1995, 102, 3110.

19 H. Meyer, J. Chem. Phys., 1995, 102, 3151.

20 M. Brouard, H. M. Lambert, S. P. Rayner and J. P. Simons, Mol. Phys., 1996, 89, 403.

21 A. J. Alexander, F. J. Aoiz, L. Baares, M. Brouard, J. Short and J. P. Simons, J. Phys. Chem., 1997, 101, 7544.

22 W. B. Chapman, M. J. Weida and D. J. Nesbitt, J. Chem. Phys., 1997, 106, 2248.

23 A. J. Orr-Ewing, W. R. Simpson, T. P. Rakitzis, S. A. Kandel and R. N. Zare, J. Chem. Phys., 1997, 106, 5961.
24 T. P. Rakitzis, S. A. Kandel, T. Lev-On and R. N. Zare, J. Chem. Phys., 1997, 107, 9392.

25 J. P. Simons, Faraday Dicuss. Chem. Soc., 1999, 113, 1.

26 T. P. Rakitzis, P. C. Samartis and T. N. Kitsopoulos, J. Chem. Phys., 1999, 111, 10415.

27 S. A. Kandel, A. J. Alexander, Z. H. Kim, R. N. Zare, F. J. Aoiz, L. Banares, J. F. Castillo and V. Sáez Rábanos, J. Chem. Phys., 2000, 112, 670 .

28 H. Meyer, in Atomic and Molecular Beams, ed. R. Campargue, Springer, Heidelberg, 2000, p. 2000.

29 J. I. Cline, K. T. Lorenz, E. A. Wade, J. W. Barr and D. W. Chandler, J. Chem. Phys., 2001, 112, 6277.

30 K. T. Lorenz, D. W. Chandler, J. W. Barr, W. Chen, G. L. Barnes and J. I. Cline, Science, 2001, 293, 2063.

31 E. A. Wade, K. T. Lorenz, D. W. Chandler, J. W. Barr, G. L. Barnes and J. I. Cline, Chem. Phys., 2004, 301, 261.

32 M. H. Alexander, J. Chem. Phys., 1999, 111, 7426.

33 M. H. Alexander, J. Chem. Phys., 1999, 111, 7435.

34 F. J. Aoiz, J. E. Verdasco, V. J. Herrero, V. Sáez Rábanos and M. H. Alexander, J. Chem. Phys., 2003, 119, 5860.

35 F. J. Aoiz, V. J. Herrero and V. Sáez Rábanos, J. Chem. Phys., 1991, 94, 7991.

36 F. J. Aoiz, V. J. Herrero and V. Sáez Rábanos, J. Chem. Phys., 1992, 97, 7423.

37 F. J. Aoiz, L. Banares and V. J. Herrero, J. Chem. Soc. Faraday Trans., 1998, 94, 2483.

38 M. H. Alexander, Chem. Phys., 1985, 92, 337.

39 R. A. LaBudde and R. B. Bernstein, J. Chem. Phys., 1971, 55, 5499.

40 M. D. Pattengill, in Atom Molecule Collision Theory, ed. R. B. Bernstein, Plenum, New York, 1979, p. 359.

41 M. P. de Miranda and F. J. Aoiz, Phys. Rev. Lett., 2004, in press.

42 R. J. Ubema, R. D. Hinchliffe and J. I. Cline, J. Chem. Phys., 1995, 103, 7934.

43 S. Althorpe, XVIII International Conference on Molecular Energy Transfer, El Escorial (Madrid), Spain, June 2003.

44 S. Bosanac, Phys. Rev. A, 1982, 26, 816.

45 U. Buck, D. Otten, R. Schinke and D. Poppe, J. Chem. Phys., 1985, 82, 202. 